\title{
Survey on Treatment Ability of Stabilization Pond as a Natural Treatment System in Oil Refinery Wastewaters
}

\author{
Abdollah Dargahi ${ }^{1}$, Mitra Mohammadi ${ }^{2}$, Amir Karami ${ }^{2}$, \\ Farhad Amirian ${ }^{3}$, Leila Tabandeh², Ali Almasi* \\ ${ }^{1}$ Department of Environmental Health Engineering, School of Public Health, Hamadan University \\ of Medical Sciences, Hamadan, Iran \\ ${ }^{2}$ Department of Environmental Health Engineering, School of Public Health, \\ Kermanshah University of Medical Sciences, Kermanshah, Iran \\ ${ }^{3}$ Department of Pathology, School of Medicine, Kermanshah University of Medical Sciences, Kermanshah, Iran
}

Received: 12 April 2017

Accepted: 22 May 2017

\begin{abstract}
An oil refinery is one industry that introduces large amounts of toxic phenol in receiving waters annually. Hence, the aim of this study was phenol removal from oil refinery wastewater by a natural treatment system of a stabilization pond. In this study, a pilot-scale pond volume, hydraulic load, and hydraulic retention time were $200 \mathrm{~L}, 40 \mathrm{~L} /$ day and five days, respectively. Influent concentration of phenol was 100-400 mg/L. Responses of the process were $\mathrm{NH}_{3}, \mathrm{PO}_{4}$, phenol, TCOD, SCOD, TBOD, SBOD, and $\mathrm{pH}$. The results showed that increasing phenol concentration and decreasing temperature have negative effects on system efficiency. Maximum removal of $\mathrm{NH}_{3}, \mathrm{PO}_{4}$, phenol, TCOD, SCOD, TBOD, and SBOD were 61.08, 70.09, 93.58, 80.18, 78.89, 78.7, and 76.84\% in high temperature, respectively. Maximum efficiency of wastewater treatment system was obtained in phenol concentration at $100 \mathrm{mg} / \mathrm{L}$. Also, anaerobic stabilization ponds are cost-effective options with simple operation that can be employed for the treatment of phenol content of oil refinery wastewaters.
\end{abstract}

Keywords: anaerobic, stabilization pond, phenol, oil refinery wastewater

\section{Introduction}

Phenol $\left(\mathrm{C}_{6} \mathrm{H}_{5} \mathrm{OH}\right)$ is one of the toxic aromatic hydrocarbons with molecular weight $94.11 \mathrm{~g} / \mathrm{mol}$. The pure solid form of phenol is white, but it is mostly colored due to the presence of impurities [1]. Phenol

*e-mail: alialmasi@yahoo.com

has a sweet taste, tar-like odour, and is soluble in organic solvents such as alcohol, glycerol, petroleum, etc. However, it is partially soluble in water [2]. Phenol is one of the most common organic pollutants due to its toxicity even at low concentrations [3-4]. Phenol and its derivatives are reported to be present in the effluent of many industries such as paper and pulp, pesticides, dyes, and chemical manufacturing [4]. Additionally, other industrial wastewaters such as resin manufacturing, gas and coke manufacturing, tanning, textiles, plastics, 
rubber, pharmaceuticals, oil refineries, ceramics, steel, coal conversion processes, phenolic resin industries, and petroleum contain different forms of phenols [5]. Phenols also are present in domestic effluents [6]. Therefore, wastewaters containing phenolic compounds can cause serious water pollution due to their poor biodegradability, high toxicity, and ecological aspects [4,7].

According to the World Health Organization (WHO), the maximum permissible concentration of phenol in potable water is $0.002 \mathrm{mg} / \mathrm{L}$. The U.S. Environmental Protection Agency (EPA) has stated that maximum permissible phenol content in wastewaters must be less than $1 \mathrm{mg} / \mathrm{L}$ [8]. Several technologies have widely been applied for phenol and phenolic compound removal from wastewaters such as physicochemical, biologica, and advanced oxidation processes (AOPs) [9-10]. But high cost, low efficiency, the formation of toxic by-products, and applicability to a limited concentration range are problems associated with the above methods. On the other hand, biological processes have an advantage over these methods which have little or no harmful effects on the environment, because these techniques do not involve the use of harmful reagents [11]. In recent years, research on various methods of biological treatment, including biodegradation of oil refinery effluents in a pond of activated sludge, was performed. Results indicated that the total chemical oxygen demand (TCOD) removal efficiency by this system was desirable [12]. Among these biological treatment sysems, wastewater stabilization ponds (WSPs) are useful for both industrial as well as municipal wastewater treatment. WSPs have been widely utilized in developing countries, especially in rural areas. WSPs offer a cheap and attractive alternative to conventional processes, in case adequate land is available. Nowadays, WSPs have been used in many parts of the world as a series of anaerobic, facultative, maturation ponds [13].

Anaerobic ponds are the smallest unit in the series. They are sized according to their volumetric organic loading, which may be in the range of 100 to $350 \mathrm{~g}$ $\mathrm{BOD}_{5} / \mathrm{m}^{3} \mathrm{~d}$, depending on the design temperature. The depth of anaerobic ponds is in the range of 2 to $5 \mathrm{~m}$ and hydraulic retention time (HRT) is usually between two and five days [14]. Anaerobic ponds work extremely well in warm climates: for example, a properly designed pond will achieve around $60 \% \mathrm{BOD}_{5}$ removal at $20^{\circ} \mathrm{C}$ and over $70 \%$ at $25^{\circ} \mathrm{C}$ and above [15]. Results of the study by Moussavi et al. showed that phenol removal efficiency using the aerobic granular SBR was more than 99\% [16]. On the other hand, Zhai's et al. study showed that using the chitosan-halloysite hybrid-nanotubes process had high phenol removal efficiency [17]. It is worth mentioning that the technology used in the above study is expensive and requires specialized experts, while the applied method in this study is the simplest and most flexible environmental technology. A literature survey revealed that there are no comprehensive reports on phenol removal from oil refinery wastewater by anaerobic WSP. Therefore, the main objective of the present study was to investigate and assess an anaerobic pond at pilot scale for treatment of Kermanshah oil refinery wastewater.

\section{Materials and Methods}

This experimental study was done as a pilot scale. The WSP with dimensions of $0.2 \times 1 \times 1 \mathrm{~m}$ were made of fiberglass plate. Experiments were carried out at ambient air temperatures ranging $25-42^{\circ} \mathrm{C}$. Average temperature of the pond was kept at $21 \pm 2^{\circ} \mathrm{C}$. In this study, HRT of the anaerobic pond was five days, and hydraulic load of this system was $40 \mathrm{~L} /$ day. The inlet of the anaerobic pond was positioned at $30 \mathrm{~cm}$ below the pond surface. The pond was loaded daily by the wastewater output of oil and grease separator unit of Kermanshah oil refinery. Before launch of the system, it underwent seeding and inoculation measures. A seeded sludge was papered by adding 1.5 liters of sewage sludge and a liter of previously prepared sludge from the oil refinery plant to the system input, before loading the system with wastewater. WSP was ready for launch after three months of seeding. The molasses was used to adjust the anaerobic pond loading besides increasing phenol concentration. In this study, phenol was added to the input of the pilot in various concentrations (100, 200, 300, and $400 \mathrm{mg} / \mathrm{L}$ ) for both warm (higher than $20^{\circ} \mathrm{C}$ ) and cold (lower than $10^{\circ} \mathrm{C}$ ) temperatures. Then the parameters of $\mathrm{NH}_{3}, \mathrm{PO}_{4}$, and phenol were measured using Varian spectropWarmometer (model UV-120-20) at wavelengths of 425, 690, and $500 \mathrm{~nm}$, respectively, and also total chemical oxygen demand (TCOD), soluble chemical oxygen demand (SCOD), total biochemical oxygen demand (TBOD), soluble biochemical oxygen demand (SBOD), and $\mathrm{pH}$ were measured.

Oxidation and reduction potential (ORP) of the pool were measured to maintain and provide anaerobic conditions. This parameter was determined using a Kent ORP meter (7020 model and with the Eil sensors).

Phenol (purity > 99.5\%) was obtained from Merck, Germany. To clarify the phenol volatility theory, the

Table 1. Characteristics of Kermanshah Oil Refinery raw wastewater.

\begin{tabular}{|c|c|}
\hline Parameter & Concentration (mg/L) \\
\hline TCOD & $622 \pm 48$ \\
\hline SCOD & $495 \pm 61$ \\
\hline TBOD & $204 \pm 16$ \\
\hline SBOD & $126 \pm 8.3$ \\
\hline TSS & $56 \pm 5.2$ \\
\hline VSS & $44 \pm 4.7$ \\
\hline N-NH3 & $13.1 \pm 2.2$ \\
\hline Phenol & $69.6 \pm 4.9$ \\
\hline pH & $7.9 \pm 0.31$ \\
\hline
\end{tabular}


pond's surface was isolated with a layer of paraffin and plastic cover and system performance was evaluated. Five consecutive samples showed that the performance rate of the anaerobic pond is almost equal in both open and closed conditions. In this study, a total of 2,400 samples were measured at two temperatures and four phenol concentrations. Descriptive statistics used for presenting data and analytical statistics by SPSS ver 21. T-test, and ANOVA were applied for comparing WSP efficiency in removal of different phenol concentrations. All sampling procedures and parameter analysis were carried out according to standard methods [18]. Table 1 shows the quality characteristics of Kermanshah oil refinery raw wastewater.

\section{Results and Discussion}

Tables 2 and 3 show the wastewater characteristics of influent and effluent of the anaerobic WSPs at high and low temperatures, and also the mean removal efficiency of measured parameters in different phenol concentrations for wastewater treatment of Kermanshah oil refinery. The operation of pilot anaerobic WSPs was provided under completely anaerobic conditions. Meeting the anaerobic conditions was confirmed by oxidation-reduction potential (ORP: -246). In the system, loading volume in warm temperatures was 104.2, 121.54, 136.01, and $148.12 \mathrm{~g} \mathrm{BOD} / \mathrm{m}^{3} \mathrm{~d}$ in phenol concentrations of 100 , 200, 300, and $400 \mathrm{mg} / \mathrm{L}$, respectively. Also, in cold temperatures the mentioned concentration was 100, $118.55,131.74$, and $143.48 \mathrm{~g} \mathrm{BOD}_{5} / \mathrm{m}^{3} \mathrm{~d}$, respectively, by considering that the standard loading volume in anaerobic ponds was in the range of $100-400 \mathrm{~g} \mathrm{BOD}_{5} / \mathrm{m}^{3} \mathrm{~d}$.

Results indicated that the studied parameters (including phenol concentration and temperature) have dramatically affected the WSP efficiency in oil refinery wastewater treatment. Therefore, the removal efficiency was significantly increased with decreasing phenol concentration and increasing temperature $(\mathrm{P}<0.001)$. According to the obtained results, the performance of a laboratory-scale anaerobic stabilization pond in removing the COD and $\mathrm{BOD}_{5}$ is better than that of Papadopoulos et al. [19]. Study results were recorded even in the worst possible conditions (high phenol concentration and low temperature). The results of the current study showed that the removal efficiencies of $\mathrm{BOD}_{5}$ and COD from the urban sewage by anaerobic WSP were $45 \%$ and $50 \%$, respectively. $\mathrm{COD} / \mathrm{BOD}_{5}$ ratio in the input and output of the system were 2.07 and 2.05, respectively. Moreover, WSP performance in the treatment of the oil refinery wastewater in different input phenol concentrations was better than that of Ghazy et al. [20] study results. In the current study, the removal efficiencies of COD, $\mathrm{BOD}_{5}$, and $\mathrm{PO}_{4}$ by WSP were $29.63 \%, 28.38 \%$, and $16.74 \%$, respectively. In our study, the increase of COD removal compared with BOD removal can be attributed to the multi-phase state of oil wastewater in which its layers have been settled on the surface and there is the volatility potential for them. Moreover, some of the layers are separated from the liquid due to hydrophobic property and precipitate in the water column of the reactor or remain suspended in the liquid column. Another advantage of this system is biodegradation of resistant materials. Due to bacterial hydrolysis, these compounds were converted into catechol, aldehydes, and acids, and can be degraded by WSP. For this reason, besides the higher removal of $\mathrm{COD}$ in the reactor effluent compared with the $\mathrm{BOD}_{5}$, the $\mathrm{COD} / \mathrm{BOD}_{5}$ ratio was identical in both input and output of the anaerobic bioreactor. These results are consistent with the Abdel-Raouf et al. study [21].

The closeness of results in our study in $\mathrm{BOD}_{5}$ and COD removal with the results of other authors like Park [15], Leven [22], and Ebrahimi [23] is evident. Their study showed that the removal efficiency of $\mathrm{BOD}_{5}$ from wastewater in anaerobic ponds under warm conditions (summer) is $60-70 \%$ and in cold conditions (winter) is $40-50 \%$. Also, it is consistent with the study undertaken by Almasi, who used the anoxic pond for wastewater treatment in both warm and cold conditions with different volumetric organic loads [24]. Almasi and Pescod [24] have shown that the rate of $\mathrm{BOD}_{5}$ and COD removal under warm conditions by the anoxic pond system were $77 \%$ and $68.28 \%$, and under the cold conditions were $62.3 \%$ and $48.95 \%$, respectively. The temperature effect on the speed of the biological reactions is an evident and proven fact. In fact at temperatures below $15^{\circ} \mathrm{C}$ an anaerobic pond acts as a sedimentation tank, which would collect plenty of the sludge [25]. The results of the Almasi et al. study showed that in domestic wastewater the COD removal efficiency decreased with decreasing temperature [26]. Moreover, the result of the Phan et al. study on phenol removal at different temperatures showed that the efficiency of the system increased with increasing temperature [27].

In a study undertaken by Ramos et al using the laboratory scale facultative stabilization pond in which wastewaters with high phenol content was used for removal of phenol with different concentrations, the results showed that the highest and lowest rates of phenol removal relates to $1,000 \mathrm{mg} / \mathrm{L}$ (92\%) and 4,000 mg/L (22\%) concentrations, respectively [28]; but for the anaerobic pond for phenol removal no independent study was found. Results of the study by Nahid et al. showed that by the increase in concentrating phenol within the range $0-200 \mathrm{mg} / \mathrm{L}$, the COD removal rate is reduced due to the phenol toxicity effect on the microbial mass activity [29]. That was in agreement with this study. The study results carried out by $\mathrm{Li}$ et al. showed that phenol removal efficiency from coal gasification wastewater by the moving bed biofilm reactor has been $89 \%$ [30]. Yousef et al. showed that by increasing the concentration of phenol in influent wastewater, the efficiency of removal by the pond is reduced [31]. The result of Yousef et al. is according to this study.

Optimal conditions resulting from this study were evaluated considering the performance of anaerobic pond in oil wastewater treatment and the decrease in the phenol concentration in the output. The highest efficiency of phenol removal in this study was obtained in a phenol 


\begin{tabular}{|c|c|c|c|c|c|c|c|c|c|c|c|c|c|}
\hline \multirow{4}{*}{ 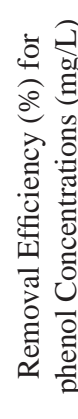 } & & 字 & ' & $\begin{array}{l}m \\
\stackrel{9}{\circ}\end{array}$ & 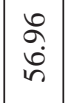 & 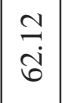 & $\begin{array}{l}\hat{\theta} \\
\infty \\
\infty \\
i n\end{array}$ & 궁 & 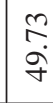 & $\stackrel{7}{\overparen{6}}$ & ' & & \\
\hline & & ৪ి & ' & กิ & \begin{tabular}{|l|} 
\\
$\infty$ \\
0 \\
$\emptyset$ \\
0
\end{tabular} & 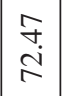 & $\begin{array}{l}\square \\
\infty \\
\infty \\
0 \\
0\end{array}$ & $\vec{g}$ & $\begin{array}{l}0 \\
\stackrel{n}{n} \\
n\end{array}$ & 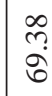 & ' & ' & \\
\hline & & 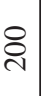 & ' & $\begin{array}{c}\tilde{m} \\
\tilde{N}\end{array}$ & 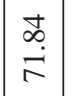 & 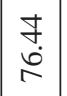 & 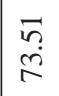 & $\begin{array}{l}\tilde{N} \\
\dot{\varphi}\end{array}$ & $\begin{array}{l}\text { नें } \\
\text { में }\end{array}$ & $\underset{\infty}{0}$ & ' & ' & \\
\hline & & 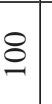 & ' & $\stackrel{\curvearrowright}{\infty}$ & \begin{tabular}{|l|} 
\\
0 \\
0 \\
0
\end{tabular} & \begin{tabular}{|l|}
$\infty$ \\
\multirow{2}{*}{} \\
$\infty$
\end{tabular} & $\begin{array}{l}\infty \\
\infty \\
\infty \\
\infty\end{array}$ & $\begin{array}{l}\infty \\
\stackrel{0}{0} \\
\dot{0}\end{array}$ & $\stackrel{8}{\stackrel{2}{0}}$ & $\begin{array}{l}\infty \\
\stackrel{\infty}{n} \\
\tilde{n}\end{array}$ & ' & ' & \\
\hline \multirow{8}{*}{ 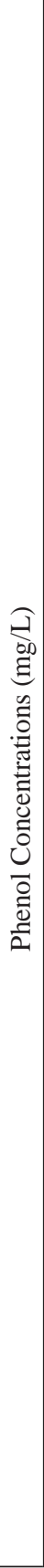 } & 8 & 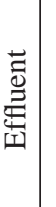 & $\begin{array}{l}\text { m. } \\
\text { †े } \\
\stackrel{N}{N} \\
\end{array}$ & 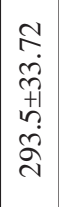 & 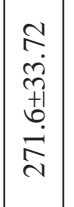 & 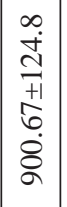 & 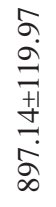 & 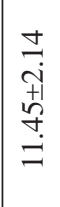 & 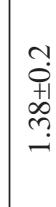 & 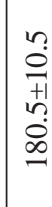 & ' & $\hat{\text { mे }}$ & \\
\hline & & 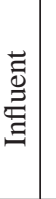 & 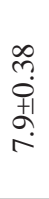 & $\begin{array}{l}-\vec{b} \\
\dot{0} \\
0 \\
01 \\
0 \\
0 \\
\\
\\
\end{array}$ & $\begin{array}{l}\vec{b} \\
\dot{0} \\
+1 \\
0 \\
0 \\
0 \\
0\end{array}$ & 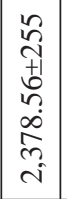 & 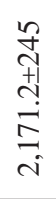 & 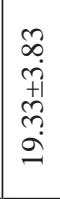 & 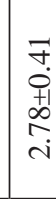 & 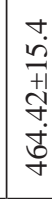 & 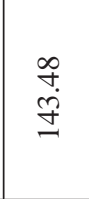 & $\vec{m}$ & \\
\hline & \multirow[b]{2}{*}{ ల్ల } & 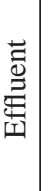 & 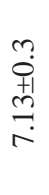 & 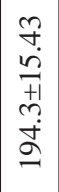 & 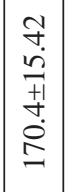 & 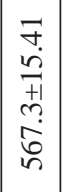 & 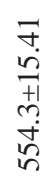 & 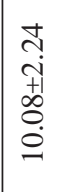 & $\begin{array}{l}\text { స̦ } \\
\text { +1 } \\
\stackrel{+1}{\sim} \\
\rightarrow\end{array}$ & 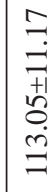 & ' & $\underset{\text { న్ }}{\tilde{\nu}}$ & \\
\hline & & $\begin{array}{l}\text { 节 } \\
\underline{\underline{\Xi}} \\
\underline{\Xi}\end{array}$ & 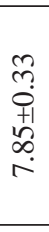 & 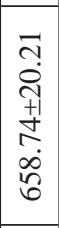 & 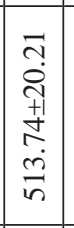 & 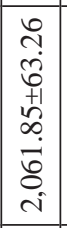 & 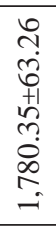 & 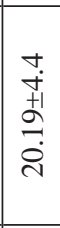 & $\begin{array}{l}+ \\
\text { ì } \\
\stackrel{+}{+} \\
\text { i }\end{array}$ & 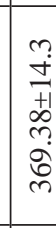 & 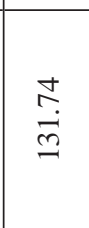 & $\stackrel{m}{m}$ & \\
\hline & & 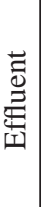 & 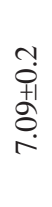 & 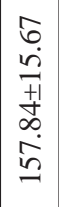 & 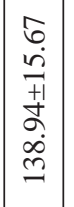 & 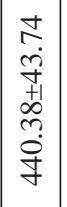 & 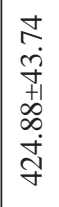 & 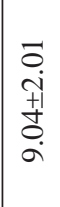 & 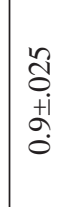 & 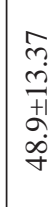 & ' & $\stackrel{\infty}{i}$ & \\
\hline & & $\begin{array}{l}\text { 节 } \\
\stackrel{\Xi}{\Xi} \\
\underline{\Xi}\end{array}$ & 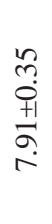 & 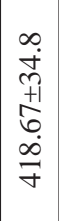 & 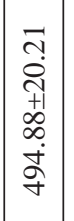 & 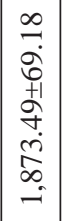 & 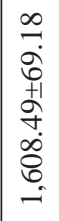 & $\begin{array}{l}\vec{\sigma} \\
\dot{m} \\
\stackrel{+}{+} \\
\stackrel{1}{0} \\
\dot{N}\end{array}$ & 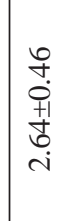 & 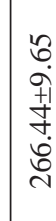 & 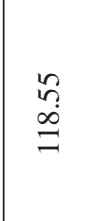 & 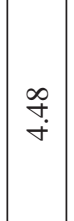 & \\
\hline & \multirow[b]{2}{*}{ 의 } & 莺 & $\begin{array}{l}m \\
0 \\
0 \\
0 \\
0 \\
\end{array}$ & \begin{tabular}{l}
$\sigma$ \\
$\dot{0}$ \\
+1 \\
+1 \\
\multirow{1}{+}{} \\
$\dot{0}$ \\
-1
\end{tabular} & $\left|\begin{array}{c}0 \\
0 \\
0 \\
+1 \\
\tilde{H} \\
\mathscr{\sigma} \\
\mathscr{\sigma}\end{array}\right|$ & 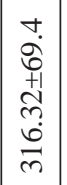 & 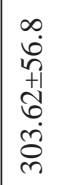 & 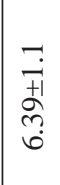 & $\begin{array}{l}1 \\
0 \\
0 \\
0 \\
0 \\
0 \\
0\end{array}$ & $\begin{array}{l}\infty \\
\stackrel{+}{+1} \\
\stackrel{0}{0} \\
\dot{\exists}\end{array}$ & ' & $\stackrel{\infty}{\stackrel{\infty}{i}}$ & \\
\hline & & 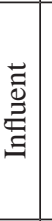 & 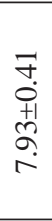 & $\begin{array}{l}\vec{b} \\
\dot{0} \\
\dot{0} \\
+1 \\
0 \\
0 \\
\dot{8} \\
\dot{n}\end{array}$ & \begin{tabular}{|c|}
$\infty$ \\
$\dot{T}$ \\
+ \\
+1 \\
0 \\
$\infty$ \\
$\infty$ \\
$\vec{\sigma}$ \\
\end{tabular} & 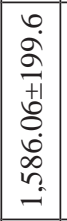 & 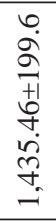 & 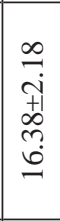 & 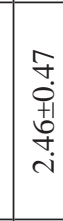 & 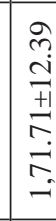 & 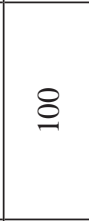 & $\underset{\stackrel{N}{N}}{\stackrel{N}{n}}$ & \\
\hline \multirow{2}{*}{\multicolumn{2}{|c|}{ 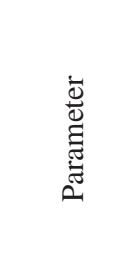 }} & & & $\begin{array}{l}0 \\
0 \\
\oplus\end{array}$ & $\begin{array}{l}0 \\
0 \\
0 \\
\omega\end{array} \mid$ & $\begin{array}{l}0 \\
0 \\
0 \\
\bullet\end{array}$ & Ô & \multirow{2}{*}{ 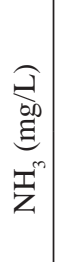 } & \multirow{2}{*}{ 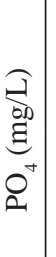 } & \multirow{2}{*}{ 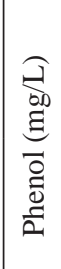 } & \multirow{2}{*}{ 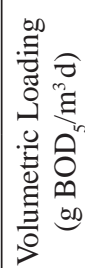 } & \multirow{2}{*}{ 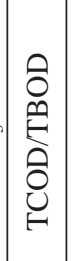 } & \\
\hline & & & & & & రి & 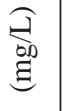 & & & & & & \\
\hline
\end{tabular}




\begin{tabular}{|c|c|c|c|c|c|c|c|c|c|c|c|c|c|}
\hline \multirow{4}{*}{\multicolumn{2}{|c|}{ 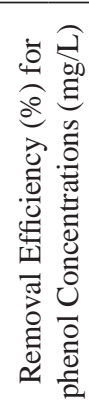 }} & \&্ণ & & $\begin{array}{l}\infty \\
\stackrel{0}{0} \\
\infty \\
i\end{array}$ & $\begin{array}{l}\vec{b} \\
\dot{\sim} \\
0\end{array}$ & $\begin{array}{l}\tilde{0} \\
\dot{N} \\
\dot{N}\end{array}$ & 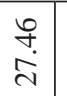 & 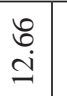 & $\begin{array}{l} \pm \\
\stackrel{\Delta}{0}\end{array}$ & $\begin{array}{l}\stackrel{\Delta}{ \pm} \\
\vec{N}\end{array}$ & & ' & \\
\hline & & ষ্লি & & $\begin{array}{l}\vec{b} \\
\dot{m} \\
\dot{m}\end{array}$ & $\begin{array}{l}\hat{\infty} \\
\dot{m} \\
\dot{m}\end{array}$ & $\begin{array}{l}\hat{0} \\
\dot{\varphi} \\
\dot{m}\end{array}$ & $\begin{array}{l}\dot{+} \\
\dot{D} \\
\dot{m}\end{array}$ & 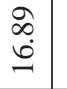 & 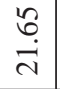 & $\begin{array}{l}0 \\
\infty \\
\dot{N}\end{array}$ & & ' & \\
\hline & & 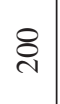 & & $\begin{array}{l}\infty \\
\infty \\
\dot{m} \\
\dot{m}\end{array}$ & $\begin{array}{l} \pm \\
0 \\
0 \\
\infty \\
m\end{array}$ & 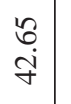 & $\begin{array}{l}\text { N̦ } \\
\stackrel{+}{0}\end{array}$ & 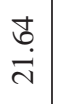 & $\begin{array}{l}\hat{h} \\
\text { ஸे } \\
\text { D }\end{array}$ & $\begin{array}{l}\text { oे } \\
\text { ம் }\end{array}$ & & ' & \\
\hline & & $\stackrel{8}{\circ}$ & & 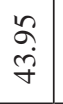 & $\begin{array}{l}\mathcal{F} \\
\vec{\forall}\end{array}$ & 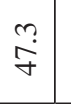 & \begin{tabular}{l}
$\widetilde{N}$ \\
\multirow{7}{*}{}
\end{tabular} & 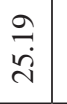 & $\begin{array}{l}\stackrel{2}{\sim} \\
\stackrel{\vec{D}}{1}\end{array}$ & $\begin{array}{l}m \\
\stackrel{\leftrightarrow}{6} \\
\dot{f}\end{array}$ & & ' & \\
\hline \multirow{8}{*}{ 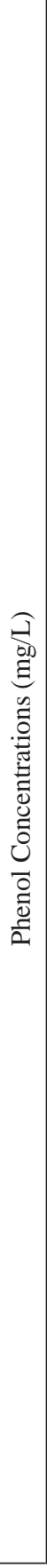 } & \multirow{2}{*}{ ஓे } & 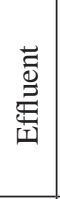 & $\begin{array}{l}\vec{m} \\
0 \\
+1 \\
\infty \\
\stackrel{n}{n}\end{array}$ & 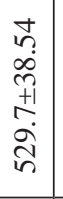 & 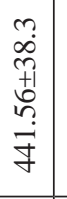 & 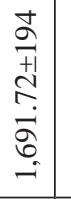 & 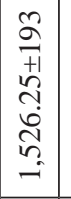 & 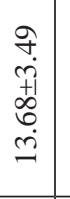 & $\begin{array}{l}0 \\
\text { m. } \\
0 \\
+1 \\
\infty \\
\stackrel{+}{-} \\
\rightarrow\end{array}$ & 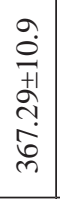 & I & $\stackrel{\vec{m}}{\vec{r}}$ & \\
\hline & & 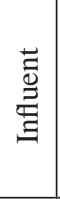 & 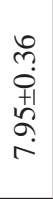 & 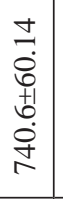 & 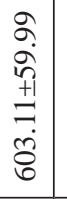 & 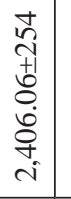 & 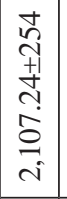 & 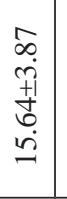 & 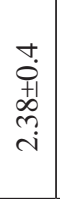 & 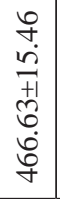 & $\begin{array}{l}\underset{7}{0} \\
\stackrel{\infty}{J}\end{array}$ & $\stackrel{\stackrel{L}{N}}{\underset{n}{n}}$ & \\
\hline & \multirow{2}{*}{ ষ্লি } & 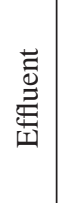 & 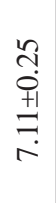 & 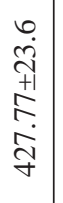 & 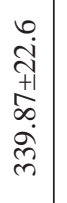 & 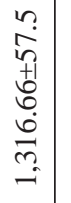 & 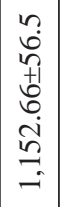 & 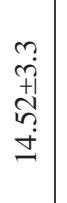 & $\begin{array}{c}0 \\
0 \\
+1 \\
⿱ 亠 䒑 \\
\stackrel{-}{-} \\
\end{array}$ & 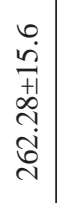 & & $\begin{array}{l}\stackrel{\infty}{0} \\
\stackrel{\text { r }}{2}\end{array}$ & \\
\hline & & $\begin{array}{l}\text { 苞 } \\
\text { 愙 }\end{array}$ & $\begin{array}{l}\text { L } \\
0 \\
0 \\
01 \\
\infty \\
\stackrel{1}{\infty}\end{array}$ & $\begin{array}{l} \pm \\
m \\
\\
\text { it } \\
+1 \\
0 \\
0 \\
\dot{0} \\
0\end{array}$ & 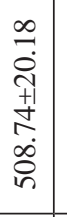 & $\begin{array}{l}\hat{0} \\
0 \\
0 \\
+1 \\
0 \\
0 \\
\dot{1} \\
0 \\
0 \\
i \\
i\end{array}$ & 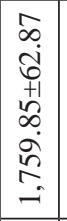 & 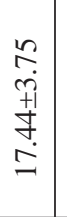 & $\begin{array}{l}\hat{\omega} \\
0 \\
+1 \\
\stackrel{1}{0} \\
\stackrel{i}{i}\end{array}$ & 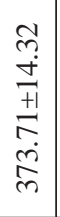 & $\begin{array}{l}\vec{\sigma} \\
\dot{\phi} \\
\stackrel{m}{\rightarrow}\end{array}$ & $\begin{array}{l}\mathscr{D} \\
\dot{m}\end{array}$ & 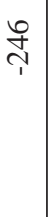 \\
\hline & \multirow{2}{*}{ 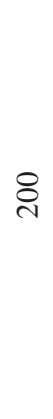 } & 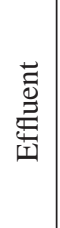 & \begin{tabular}{l}
$\hat{N}$ \\
$o$ \\
I \\
\multirow{N}{N}{}
\end{tabular} & 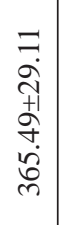 & 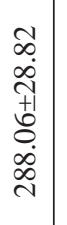 & 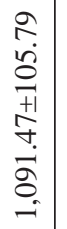 & 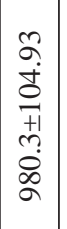 & $\begin{array}{l}\stackrel{O}{+} \\
i \\
+1 \\
\stackrel{0}{0} \\
i\end{array}$ & 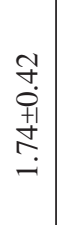 & 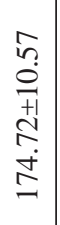 & & 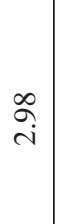 & \\
\hline & & 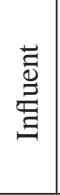 & 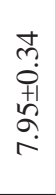 & 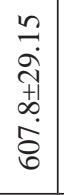 & 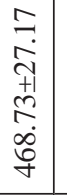 & 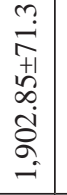 & 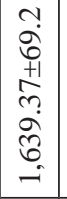 & 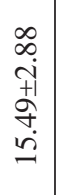 & 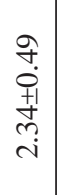 & 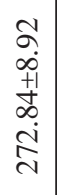 & 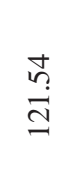 & $\stackrel{m}{\vec{m}}$ & \\
\hline & \multirow{2}{*}{$\stackrel{8}{9}$} & 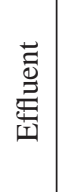 & $\begin{array}{l}\vec{m} \\
0 \\
+1 \\
0 \\
0 \\
\end{array}$ & 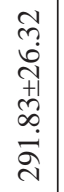 & 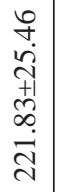 & 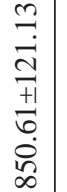 & 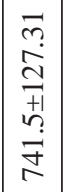 & 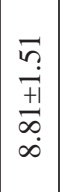 & $\begin{array}{l}\stackrel{L}{\sim} \\
0 \\
+1 \\
\stackrel{1}{0} \\
\stackrel{\leftrightarrow}{\rightarrow} \\
-\end{array}$ & 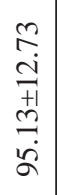 & ' & ๙ે & \\
\hline & & 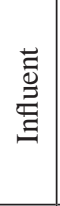 & 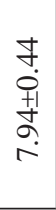 & 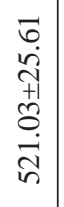 & 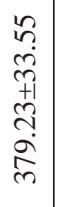 & 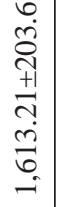 & 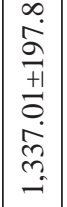 & 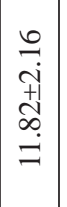 & 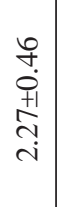 & 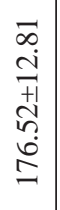 & $\underset{\sim}{\stackrel{N}{+}}$ & $\underset{\text { iे }}{\stackrel{8}{1}}$ & \\
\hline \multicolumn{2}{|r|}{ 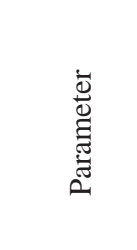 } & & $\bar{a}$ & 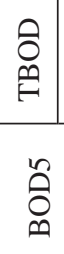 & $\begin{array}{l}0 \\
\text { की } \\
\text { n. }\end{array}$ & $\begin{array}{l}\text { Oे } \\
\text { }\end{array}$ & 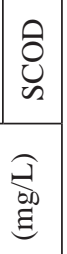 & 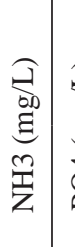 & 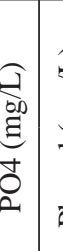 & 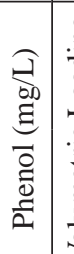 & 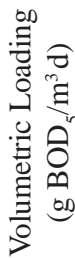 & 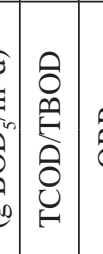 & حิ वे \\
\hline
\end{tabular}


concentration of $100 \mathrm{mg} / \mathrm{L}$ (93.58\%) after five days and warm weather, which is more than phenol removal by the $\mathrm{UV} / \mathrm{TiO}_{2}$ process and less than the RBC biological system. Considering the fact that the anaerobic ponds in all different phenol concentrations (except $100 \mathrm{mg} / \mathrm{L}$ ) is not by itself capable of removing the organic pollutants up to permissible standards of discharge into the environment, it must be employed as pre-treatment and subsequently the anoxic and facultative stabilization pond must be used.

The results showed that in cold conditions the efficiency of anaerobic pond in oil wastewater treatment with different concentrations of phenol is relatively low. This can be related to the low growth activity of microorganisms and slow reaction rate of decomposition of dissolved materials by them. Besides, phenol as part of organic compounds forming $\mathrm{BOD}_{5}$ and $\mathrm{COD}$ is dissolved into solution and lacks the potential of sedimentation in an anaerobic pond; this finding is consistent with Almasi and Pescod [24]. On the whole it can be concluded that anaerobic stabilization ponds, if properly operated, show favorable performance in removing the organic compounds at different concentrations of phenol in warm temperatures.

\section{Conclusions}

Just as seen in the results, maximum removal of $\mathrm{NH}_{3}$, $\mathrm{PO}_{4}$, phenol, TCOD, SCOD, TBOD, and SBOD were 61.08, 70.09, 93.58, 80.18, 78.89, 78.7, and 76.84\%, respectively. That was obtained in phenol concentration of $100 \mathrm{mg} / \mathrm{L}$ and high temperature. Climate conditions affected pond treatment performance so that system efficiency was minimum at low temperature. Considering the advantages of a WSP system such as flexibility, simplicity of operation, and relatively high efficiency, it can be used as a better alternative in comparison of other expensive and complex systems. Since the WSP removal efficiency for phenol and phenolic compounds was better in comparison with conventional biological treatment methods, it can be concluded that anaerobic pond systems as an option with proper cost- effectiveness can be employed for treatment of petrochemicals and oil refinery phenolic wastewaters.

\section{Acknowledgements}

This work was supported by the Kermanshah University of Medical Sciences.

\section{References}

1. SHOKOOHI R., MOVAHEDIAN H., DARGAHI A. Evaluation of the Efficiency of a Biofilter System's Phenol Removal From Wastewater. Avicenna Journal of Environmental Health Engineering. 3, 1, 2016.
2. BASHA K.M., RAJENDRAN A., THANGAVELU V. Recent advances in the biodegradation of phenol: a review. Asian J Exp Biol Sci. 1, 219, 2010.

3. DARGAHI A., ALMASI A., MAHMOODI M., KHAMOOTIAN R. Performance of the facultative stabilization pond in removing phenol from oil refinery effluent. J. of Water and Wastewater. 8, 25, 2012.

4. BU Q., LEI H., REN S., WANG J., HOLLADAY J., ZHNG Q., et al. Phenol and phenolics from lignocellulosic biomass by catalytic microwave pyrolysis. Bioresource technology. 102, 7004, 2011.

5. DARGAHI A., ALMASI A., SOLTANIAN M., ZAREI P., HASHEMIAN A., GOLESTANIFAR H. Effect of Molasses on Phenol Removal Rate Using Pilot-Scale Anaerobic Reactors. J Water Wastewater. 25, 3, 2014.

6. CAETANO M., VALDERRAMA C., FARRAN A., CORTINA J.L. Phenol removal from aqueous solution by adsorption and ion exchange mechanisms onto polymeric resins. Journal of Colloid and Interface Science. 338, 402, 2009.

7. ASLAM M., QAMAR M., SOOMRO M. T., ISMAEIL M.,SALAH N., ALMEELBI T., et al. The effect of sunlight induced surface defects on the photocatalytic activity of nanosized $\mathrm{CeO} 2$ for the degradation of phenol and its derivatives. Applied Catalysis B: Environmental. 108, 391, 2016.

8. GANDHI G.R., IGNACIMUTHU M., PAULRAJ M.G. Solanum torvum Swartz. fruit containing phenolic compounds shows antidiabetic and antioxidant effects in streptozotocin induced diabetic rats. Food and Chemical Toxicology. 49, 2725, 2011.

9. XU L., WANG J. A heterogeneous Fenton-like system with nanoparticulate zero-valent iron for removal of 4-chloro-3methyl phenol. Journal of hazardous materials. 186, 256, 2011.

10. DARGAHI A., SHARAFI K., ALMASI A., ASADI F., et al. Effect of biodegradable organic matter concentration on phenol removal rate from oil refinery wastewater using anaerobic pond system. Journal of Kermanshah University of Medical Sciences (J Kermanshah Univ Med Sci). 18, 690, 2015.

11. JARBOUI R., CHTOUROU M., AZRI C., GHARSALLAH N., AMMAR E. Time-dependent evolution of olive mill wastewater sludge organic and inorganic components and resident microbiota in multi-pond evaporation system. Bioresource technology. 101, 5749, 2010.

12. MISTI T., TEZEL U., Pavlostathis S.G. Fate and effect of naphthenic acids on oil refinery activated sludge wastewater treatment systems. Water research. 47, 449, 2013.

13. MBURU N., TEBITENDAW S.M., VAN BRUGGEN J.J., ROUSSEAUD P., LENS P.N. Performance comparison and economics analysis of waste stabilization ponds and horizontal subsurface flow constructed wetlands treating domestic wastewater: A case study of the Juja sewage treatment works. Journal of environmental management. 128, 220, 2013.

14. MARTIN J., SANTOS J.L., APARICIO L., ALONOSO E. Pharmaceutically active compounds in sludge stabilization treatments: Anaerobic and aerobic digestion, wastewater stabilization ponds and composting. Science of The Total Environment. 503, 97, 2015.

15. PARK J., CRAGGAS R., SHILTON. Wastewater treatment high rate algal ponds for biofuel production. Bioresource technology. 102, 35, 2011.

16. MOUSSAVI G., BARIKBIN B., MAHMOUDI M. The removal of high concentrations of phenol from saline wastewater using aerobic granular SBR. Chemical Engineering Journal. 158, 498, 2013. 
17. ZHAI R., ZHANG B., WAN Y., LI C., WANG J., LIU J. Chitosan-halloysite hybrid-nanotubes: Horseradish peroxidase immobilization and applications in phenol removal. Chemical engineering journal. 214, 304, 2013.

18. APHA. Standard Methods for the Examination for Water and Wastewater, 21 ${ }^{\text {th }}$ Ed. American Public Health Association, Washington, DC., USA, 2005.

19. PAPADOUPOULOS A., PARISSOPOULOS G., PAPADOUPOULOS F., KARTERIS A. Variations of COD/BOD ratio at different units of a wastewater stabilization pond pilot treatment facility. in Proceeding of $7^{\text {th }}$ International Conference on Environmental Science and Technology Ermoupolis. 2001.

20. GHAZY M.M.E.D., SENOUSY W.M.E., AATTAY A.M.A., KAMEL M. Performance evaluation of a waste stabilization pond in a rural area in Egypt. American Journal of Environmental Sciences. 4, 316, 2008.

21. ABDEL-RAOUF N., A. Al-HOMAIDAN I. Microalgae and wastewater treatment. Saudi Journal of Biological Sciences. 19, 257, 2012.

22. LEVEN L., NYBERG K., SCHNURER K. Conversion of phenols during anaerobic digestion of organic solid waste - a review of important microorganisms and impact of temperature. Journal of environmental management. 95, 99, 2012.

23. EBRAHIMI A., EHRAMPOOSH M. H., SHAHSAVARI E., HOSSEINI E., HASHEMI H., TALAEI P., et al. Survey on removal efficiency of linear alkylbenzene sulfonate in Yazd stabilization pond. International Journal of Environmental Health Engineering. 4, 10, 2015.

24. ALMASI A., PESCOD M. Wastewater treatment mechanisms in anoxic stabilization ponds. Water Science and Technology. 33, 125, 1996.
25. MENGESHA D. Performance evaluation of kality wastewater stabilisation pond for the treatment of municipal sawage. 34, 71, 2010.

26. ALMASI A., SHARAFI K., HAZRATI S., FAZLZADEH DAVIL M., et al. A survey on the ratio of effluent algal BOD concentration in primary and secondary facultative ponds to influent raw BOD concentration. Desalination and Water Treatment. 53, 3475, 2015.

27. PHAN-VAN M., ROUSSEAU D., DE PAUW N. Effects of fish bioturbation on the vertical distribution of water temperature and dissolved oxygen in a fish culture-integrated waste stabilization pond system in Vietnam. Aquaculture. 281, 28, 2008.

28. RAMOS M., DAVILA J., ESPARZA F., THALASSO F., ALBA J., GUERREO A., et al. Treatment of wastewater containing high phenol concentrations using stabilisation ponds enriched with activated sludge. Water Science and Technology. 51, 257, 2005.

29. NAHID P., KAZEMI A. The optimization of microorganisms activities in the Tehran oil refinery biological wastewater treatment. J. of Water and Wastewater. 50, 23, 2004.

30. LI H.Q., LI H.Q., HAN H.J., DU M.A., WANG W. Removal of phenols, thiocyanate and ammonium from coal gasification wastewater using moving bed biofilm reactor. Bioresource technology. 102, 4667, 2011.

31. YOUSEFI R.I., El-ESWED B., ALA'A H. Adsorption characteristics of natural zeolites as solid adsorbents for phenol removal from aqueous solutions: kinetics, mechanism, and thermodynamics studies. Chemical Engineering Journal. 171, 1143, 2011. 